\title{
Common Genetic Variation in the Promoter of the Human apo CIII Gene Abolishes Regulation by Insulin and May Contribute to Hypertriglyceridemia
}

\author{
W. William Li, * Marilyn M. Dammerman, ${ }^{\ddagger}$ Jonathan D. Smith, ${ }^{\ddagger}$ Shula Metzger, Jan L. Breslow, ${ }^{\ddagger}$ and Todd Leff \\ *Department of Signal Transduction, Parke-Davis Co., and the Department of Biological Chemistry, University of Michigan Medical \\ School, Ann Arbor, Michigan 48105; and ${ }^{\ddagger}$ Laboratory of Biochemical Genetics and Metabolism, The Rockefeller University, \\ New York 10021
}

\begin{abstract}
Overexpression of plasma apolipoprotein CIII (apo CIII) causes hypertriglyceridemia in transgenic mice. A genetically variant form of the human apo CIII promoter, containing five single base pair changes, has been shown to be associated with severe hypertriglyceridemia in a patient population. In animals and in cultured cells the apo CIII gene is transcriptionally downregulated by insulin. In this study we demonstrate that, unlike the wild-type promoter, the variant promoter was defective in its response to insulin treatment, remaining constitutively active at all concentrations of insulin. The loss of insulin regulation was mapped to polymorphic sites at $-\mathbf{4 8 2}$ and $-\mathbf{4 5 5}$, which fall within a previously identified insulin response element. Loss of insulin regulation could result in overexpression of the apo CIII gene and contribute to the development of hypertriglyceridemia. The variant apo CIII promoter is common in the human population and may represent a major contributing factor to the development of hypertriglyceridemia. (J. Clin. Invest. 1995. 96:2601-2605.) Key words: transcription, genetic - gene expression regulation - hypertriglyceridemia • apolipoproteins $\mathbf{C} \cdot$ insulin resistance
\end{abstract}

\section{Introduction}

apo CIII is a constituent of triglyceride (TG) ${ }^{1}$ rich lipoproteins and is an important regulator of plasma TG concentrations (1, 2 ). Studies in transgenic mice have demonstrated that the level of apo CIII has a direct and dramatic influence on plasma TG levels $(3,4)$. As little as $40 \%$ additional apo CIII results in more than a doubling of plasma TGs. Related findings have been reported in clinical studies in which apo CIII levels have been observed to be correlated with elevated plasma TG levels and delayed clearance of TG-rich lipoproteins (5-7). Finally,

Address correspondence to Todd Leff, Signal Transduction, Parke-Davis Co., 2800 Plymouth Rd., Ann Arbor, MI 48105. Phone: 313-998-5989; FAX: 313-998-5970; e-mail: lefft@aa.wl.com Shula Metzger’s current address is Hadassah Medical School, Hebrew University, Jerusalem, Israel.

Received for publication 26 June 1995 and accepted in revised form 21 August 1995.

1. Abbreviations used in this paper: CAT, chloramphenicol acetyltransferase; TG, triglyceride.

J. Clin. Invest.

(c) The American Society for Clinical Investigation, Inc.

0021-9738/95/12/2601/05 $\$ 2.00$

Volume 96, December 1995, 2601-2605 genetic studies have demonstrated a strong association of a polymorphism in the $3^{\prime}$ UTR of the human apo CIII gene with hypertriglyceridemia in several distinct populations (8-12). Taken together, these studies strongly suggest that expression of the apo CIII gene is an important determinant of plasma TG levels in the human population.

The regulation of apo CIII gene expression appears to occur primarily at the level of transcription. In liver cells, the transcriptional activity of the gene is regulated by a variety of agents, including inflammatory cytokines like IL-1 (13) and hypolipidemic agents such as fibric acids (14). In addition, we have demonstrated recently that the apo CIII gene is regulated by insulin in animals and in cultured hepatocytes (15). In these studies, treatment of insulin-deficient diabetic mice with insulin resulted in a 2.5-fold decrease in hepatic apo CIII mRNA levels and a corresponding decrease in apo CIII gene transcriptional activity. In addition, it was shown that insulin treatment of HepG2 cells transfected with an apo CIII luciferase reporter construction caused a dose-dependent twofold reduction in apo CIII transcriptional activity.

Recently, the promoter of the human apo CIII gene was found to be genetically polymorphic, containing five sites of single base pair sequence variation between -641 and -455 (Fig. 1). In an association study, a haplotype of the CIII gene containing all five sequence changes was found to be associated with an increased risk of hypertriglyceridemia (16). One possible explanation for the association of the variant promoter with elevated triglycerides is that one or more of these changes increases the transcriptional activity of the apo CIII gene, thereby causing an increase in apo CIII levels and inducing the development of hypertriglyceridemia. The goal of the current study is to determine if the DNA sequence changes that occur in the variant promoter cause a change in the transcriptional activity of the apo CIII gene.

\section{Methods}

Plasmids. CAT expression vectors were derived from the puc 18-based plasmid pKTCIII, which contains the bacterial chloramphenicol acetyltransferase (CAT) gene under the control of the human apo CIII promoter (17). A derivative of pKTCIII containing apo CIII sequences -854 to +22 from a human genomic library served as the wild-type (11111) construction. The 22222 construction was derived from a cloned PCR amplification product from genomic DNA of a subject with the 22222 variant. The 22211 and 11122 constructions were made by exchanging an upstream or downstream restriction fragment cassette between the 11111 and 22222 parent constructions. Luciferase expression vectors were derived directly from the apo CIII/CAT vectors by removal of the KpnI-HindIII fragment containing apo CIII promoter sequences $(-854$ to +22$)$ from the 11111, 22222, 11122, and 22211 constructions. These fragments were then inserted upstream of the luciferase coding sequences in the reporter vector pGL2-basic (Promega, Madison, WI). Construction of 11112 and 11121 promoters was carried 
A

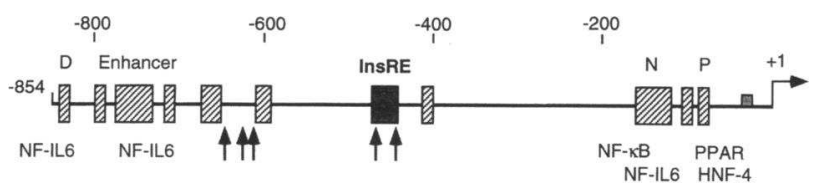

B

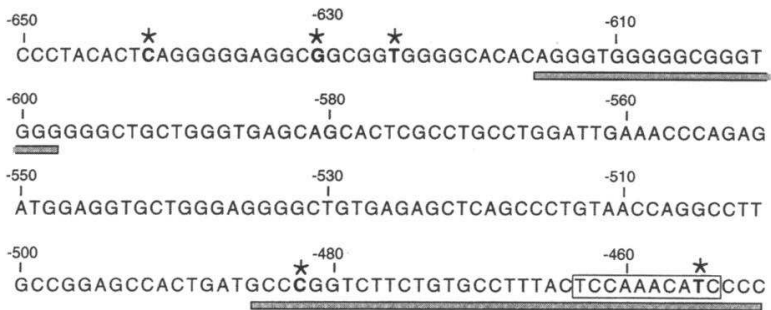

C

\begin{tabular}{|c|c|c|c|c|c|}
\hline & -641 & -630 & -625 & -482 & -455 \\
\hline Allele & 1 & 1 & 1 & 1 & 1 \\
\hline 11111 & C & G & $\mathrm{T}$ & C & $T$ \\
\hline 22222 & $A$ & A & - & $\mathrm{T}$ & C \\
\hline
\end{tabular}

Figure 1. Sequence variation in the human apo CIII promoter. (A) A schematic representation of the human apo CIII promoter. Hatched boxes (labeled above) indicate regions that have been shown to interact with DNA binding proteins and in some cases have been shown to have specific transcriptional activity $(13,17,18,25)$. Known transcription factor interactions are indicated below the line. Arrows indicate location of the five single base pair polymorphic sites. $(B)$ DNA sequence of the region of the apo CIII promoter containing the five polymorphic sites (indicated by asterisks). Shaded bars indicate footprinted regions $(-616 /-598,-485 /-452)$. The functional insulin response element (C3IRE) has been mapped to -490 to -449 ( $\mathrm{Li}, \mathrm{W}$. W., and T. Leff, manuscript in preparation). The boxed nucleotides indicate a sequence similar to the rat PEPCK insulin response element $(15,26)$. (C) The sequence configuration of the most common CIII promoter allele (11111) and the second most common allele (22222) containing single nucleotide changes at each of the five sites (16).

out by introduction of single base pair changes into the wild-type (11111) promoter, using a site-directed mutagenesis kit (Bio Rad Labs., Hercules, CA). All constructions were verified by DNA sequencing.

Transfections. Transfection of the apo CIII/CAT expression vectors into HepG2 cells was carried out as described (18). Transfection efficiency was determined by cotransfection of a $\beta$-galactosidase-expressing reference plasmid. apo $\mathrm{CIII/luciferase} \mathrm{constructions} \mathrm{were}$ transfected into HepG2 cells in $20 \%$ confluent 48 -well plates using lipofectin according to the manufacturer's protocol (GIBCO BRL, Gaithersburg, MD) with $1.375 \mu \mathrm{g}$ of DNA per well. Cells were transfected for $4 \mathrm{~h}$ and allowed to recover in MEM plus $10 \%$ fetal bovine serum for $48 \mathrm{~h}$, followed by insulin treatment for $6 \mathrm{~h}$ in serumfree media. Cells were harvested and luciferase activity was measured and normalized to total protein as described (15). Experiments with insulin treatment were carried out in the absence of a $\beta$-gal internal reference plasmid. Statistical significance was calculated using the Student's two-tailed $t$ test.

Gel shifts. Nuclear extracts were prepared from liver isolated from hyperglycemic female Sprague-Dawley rats. Hyperglycemia was in-
Table I. Basal Transcriptional Activity of apo CIII Promoter Variants

\begin{tabular}{lcccc}
\hline & \multicolumn{4}{c}{ Promoter genotype } \\
\cline { 2 - 5 } & 11111 & 11122 & 22211 & 22222 \\
\hline $\begin{array}{c}\text { Relative CAT activity } \\
( \pm S D)\end{array}$ & 1.00 & 1.17 & 0.96 & 1.09 \\
$( \pm 0.09)$ & $( \pm 0.18)^{*}$ & $( \pm 0.16)$ & $( \pm 0.32)$
\end{tabular}

CAT activity (normalized to reference $\beta$-gal activity) from different apo CIII promoter variants transfected into HepG2 cells, expressed relative to the activity of the wild-type (11111) construction. Values are the means of seven independent experiments performed in triplicate ( $n$ $=21)$. ${ }^{*}$ Significant difference from the wild-type (11111) construction $(P<0.0005)$.

duced by a $65 \mathrm{mg} / \mathrm{kg}$ intravenous injection of streptozotocin $12 \mathrm{~d}$ before time of killing. Liver tissue was disrupted in a Teflon glass homogenizer, and the cells were lysed in hypotonic buffer. Nuclei were extracted essentially as described (19). Gel mobility shift assay was performed as described (17), in the presence of $0.5 \mathrm{mM} \mathrm{MgCl}_{2}, 0.25 \mathrm{ng}$ labeled probe $(50,000 \mathrm{cpm})$, and $9 \mu \mathrm{g}$ of nuclear extract. Gels were analyzed on a Molecular Dynamics PhosphorImager (Sunnyvale, CA).

\section{Results and Discussion}

The DNA sequences of the wild-type and variant versions of the apo CIII promoter are shown in Fig. 1. To determine if the sequence changes in the variant promoter affect apo CIII transcriptional activity, we constructed a series of apo CIII promoter/CAT reporter plasmids containing various versions of the apo CIII promoter. Reporter constructions containing either the wild-type promoter (11111), the variant promoter (22222), or one of two in vitro generated promoters (11122 and 22211) were transfected into HepG2 cells and the relative CAT activity was measured. The results (Table I) demonstrate that all four promoters showed approximately the same activity, although the promoter containing the variant sequence at the two downstream sites (11122) was expressed at a slightly, but significantly, higher level than wild-type (11111).

Although basal activity was not strongly affected, it remained a possibility that one or more of these changes affected the ability of the promoter to respond to cellular signals that modulate apo CIII transcription. Two of the five sites of sequence variation (at positions -482 and -455 ) fall within a region that has been characterized recently by mutational analysis as a functional insulin response element $(\mathrm{Li}, \mathrm{W}$. W., and T. Leff, manuscript in preparation). In addition, the -455 site is within a sequence that is similar to an insulin response element found in the rat PEPCK gene promoter (Fig. $1 B$ ). These observations suggested that the variant promoter might be defective in its ability to be downregulated by insulin. To test this possibility, we transfected a series of apo CIII promoter/luciferase reporter constructs into HepG2 cells subsequently treated with increasing concentrations of insulin. The wild-type promoter (11111) and the recombinant promoter containing the wildtype sequence at the two downstream sites (22211) were downregulated approximately twofold by insulin in a dose-dependent manner (Fig. 2). The degree of downregulation was consistent with that observed in animal studies (15). In contrast, both the variant $(22222)$ promoter and the recombinant promoter 
A

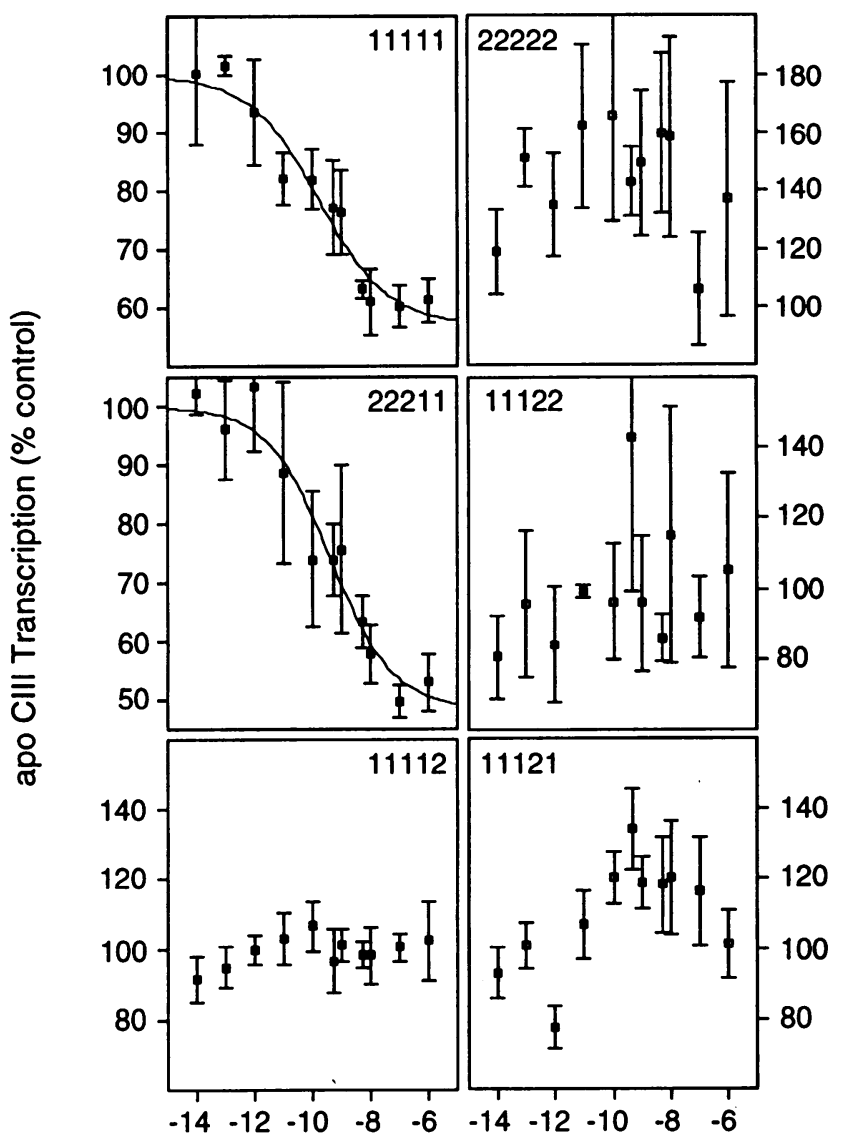

Log insulin concentration (M)
B

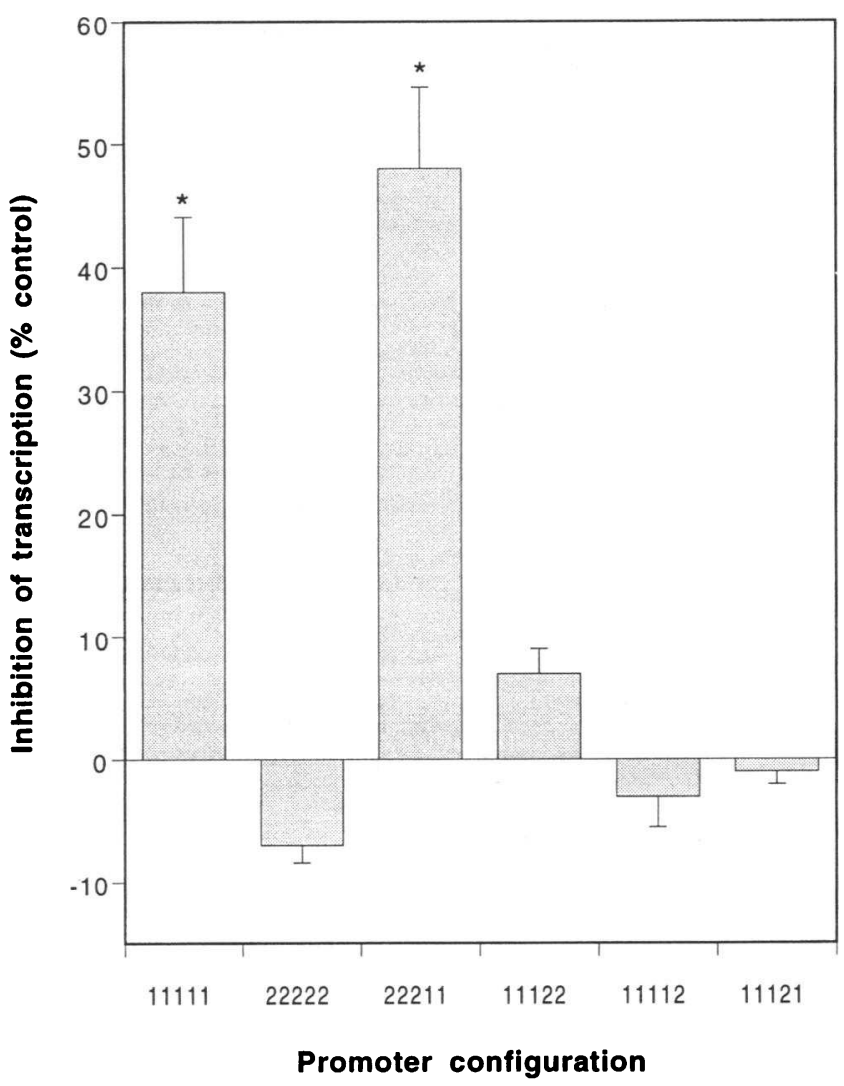

Figure 2. Regulation of apo CIII promoter variants by insulin. $(A)$ Relative transcriptional activity of wild-type and variant promoter-reporter constructs transfected into HepG2 cells in the presence of different concentrations of insulin. The genotype of the promoter is indicated in the upper corner of each panel according to Fig. $1 C$. Data are presented as the percentage of the mean of the vehicle (no-insulin) control. Each point represents the mean of 6-12 replicates, \pm standard error. $(B)$ Comparison of the effect of $1 \mu \mathrm{M}$ insulin on the transcriptional activity of each apo CIII promoter configuration. Data are presented as percent repression of transcriptional activity relative to the vehicle (no-insulin) control for each construction. Each point represents the mean of at least three independent experiments (4-12 replicates each) \pm standard error. The asterisk indicates significant difference between transcriptional activity in insulin versus vehicle $(P<0.005)$.

containing the variant sequence at the two downstream sites (11122) were defective in their response to insulin treatment, remaining at elevated levels at all doses of insulin (Fig. 2), indicating that the defective insulin response is due to changes at the two downstream sites. To determine if variation at one or both of these sites is responsible for the loss of insulin regulation, each was individually reconstructed into the wild-type promoter background and transfected into HepG2 cells. Neither the 11121 nor the 11112 construction was responsive to insulin treatment (Fig. 2), demonstrating that a single base pair change at either the -482 site or the -455 site is sufficient to abolish the insulin responsiveness of the apo CIII promoter.

These findings suggest that a subject with a variant promoter would have an apo CIII gene that was not properly downregulated by insulin. In an otherwise insulin-responsive subject, this would result in a relative overexpression of the apo CIII gene, leading to increased susceptibility to the development of hypertriglyceridemia. It may be significant that several of the variant constructions show a mild dose-dependent stimulation of tran- scription by insulin (22222, 11122, and 11121, Fig. $2 A$ ) and increased basal transcriptional activity (11122, Table I). These changes could further increase apo CIII production in patients containing a variant allele. The possibility that the variant promoter configuration could lead to overexpression of the apo CIII gene and a tendency to develop hypertriglyceridemia is consistent with results from a previous genetic association study (16). In this study apo CIII alleles consisting of the 22222 or 22212 promoter variants, and the less common (S2) allele of a polymorphic SstI site in the 3' UTR of the apo CIII gene, were associated with an increased risk of hypertriglyceridemia. These findings suggest that, in at least some genetic backgrounds, the variant apo CIII promoter may contribute to the development of hypertriglyceridemia.

A possible explanation for the loss of insulin regulation by the variant promoter is a reduced affinity for the transcription factors that mediate the insulin response. To test this possibility, we compared the ability of the wild-type and variant versions of the insulin response element (C3IRE; -485 to -450 ) to 


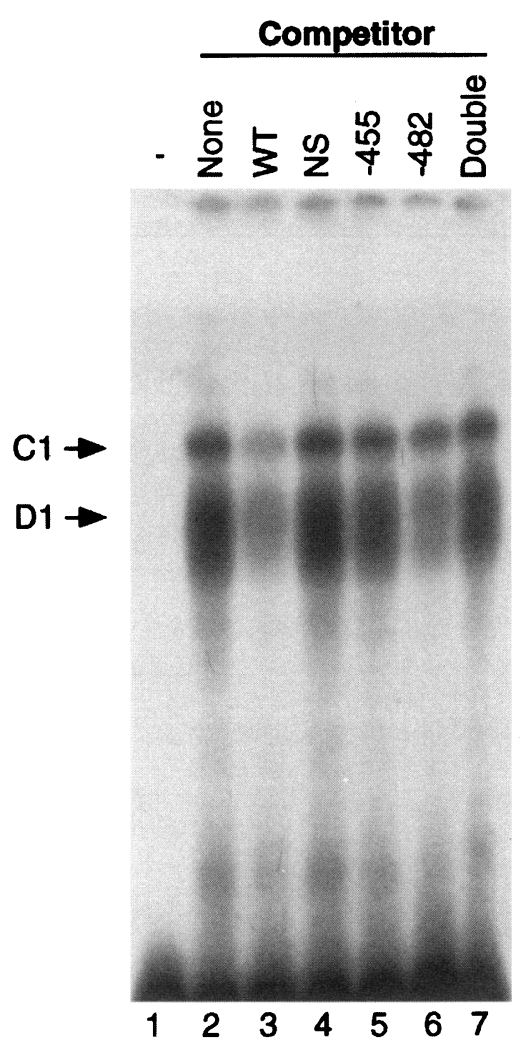

two primary DNA binding protein activities in this extract (which was prepared from hyperglycemic animals), $D l$ and $C l$, are indicated. Similar results were obtained in experiments carried out with rat liver nuclear extracts prepared from normal animals; except that these extracts do not contain the D1 binding activity (data not shown).

interact with specific DNA binding proteins in rat liver nuclear extracts. As presented in Fig. 3, two DNA binding activities interacted with an oligonucleotide probe representing the C3IRE. The binding of these two proteins could be completed by the wild-type oligonucleotide ( self-competition), but not by the same amount of a variant oligonucleotide containing either a single base pair change at -455 or the double change ( -455 and -482) (Fig. 3, lanes 3, 5, and 7). In contrast, the C3IRE oligonucleotide containing the single base pair change at -482 was nearly as good a competitor as the wild-type oligonucleotide (Fig. 3, lanes 3 and 6), indicating that the change at -482 did not affect the binding of these two proteins to the C3IRE. This was an unexpected result in light of the clear transcriptional effect of a sequence change at the -482 site (Fig. 2). It is possible that the transcription factors interacting with sequences around the -482 site in vivo are not present in our nuclear extract preparations. It should be noted that, in the human population, the -482 sequence variant rarely appears without the -455 sequence variant (16). For this reason the in vitro behavior of the isolated -482 mutation is of limited significance with regard to the role of the variant promoter in human physiology. Taken together, these results demonstrate that a single base pair change at the -455 site reduces the affinity of the C3IRE for specific DNA binding proteins, and provides a potential explanation for the inability of the variant apo CIII promoter allele to respond to insulin.

Several instances of inherited defects in transcriptional regu- lation have been described (for examples see references 20 23 ). The variation in the promoter of the apo CIII gene is, to our knowledge, the first example of a genetic polymorphism in an insulin response element. The defective apo CIII insulin response element can be considered an example of insulin resistance at the level of the gene. Although overexpression of human apo CIII does not cause diabetes in transgenic mice (24), the paradigm of insulin resistance at the transcriptional level, as exemplified by apo CIII promoter, may prove applicable to other genes. Insulin-resistant transcriptional regulation of genes directly involved in glucose metabolism could potentially play a role in the development of general insulin resistance and noninsulin-dependent diabetes mellitus.

\section{Acknowledgments}

We thank Edmund Wong and Jeffrey Halaas for technical assistance.

This work was supported by grants from the National Institutes of Health (HL-45394 to T. Leff and HL-33714 and HL-32435 to J. L. Breslow) and the American Heart Association and the American Heart Association New York City Affiliate to M. M. Dammerman.

\section{References}

1. Havel, R. P., and J. P. Kane. 1995. Structure and metabolism of plasma lipoproteins. In The Metabolic and Molecular Basis of Inherited Disease. R. C. Scriver, A. L. Beaudet, W. S. Sly, and D. Valle, editors. McGraw-Hill, Inc., New York. 1841-1852.

2. Breslow, J. L. 1993. Genetics of lipoprotein disorders. Circulation. 87:1621.

3. Ito, Y., N. Azrolan, A. O'Connell, A. Walsh, and J. L. Breslow. 1990. Hypertriglyceridemia as a result of human apo CIII gene expression in transgenic mice. Science (Wash. DC). 249:790-793.

4. Aalto-Setäla, K., E. A. Fisher, X. Chen, T. Chajek-Shaul, T. Hayek, R. Zechner, A. Walsh, R. Ramakrishnan, H. N. Ginsberg, and J. L. Breslow. 1992. Mechanism of hypertriglyceridemia in human apolipoprotein (apo) CIII transgenic mice. Diminished very low density lipoprotein fractional catabolic rate associated with increased apo CIII and reduced apo E on the particles. J. Clin. Invest. 90:1889-1900.

5. Carlson, L. A., and D. Ballantyne. 1976. Changing relative proportions of apolipoproteins CII and CIII of very low density lipoproteins in hypertriglyceridaemia. Atherosclerosis. 23:563-568.

6. Ginsberg, H. N., N. A. Le, I. J. Goldberg, J. C. Gibson, A. Rubinstein, P. Wang-Iverson, R. Norum, and W. V. Brown. 1986. Apolipoprotein B metabolism in subjects with deficiency of apolipoproteins CIII and AI. Evidence that apolipoprotein CIII inhibits catabolism of triglyceride-rich lipoproteins by lipoprotein lipase in vivo. J. Clin. Invest. 78:1287-1295.

7. Malmendier, C. L., J. F. Lontie, C. Delcroix, D. Y. Dubois, T. Magot, and L. De Roy. 1989. Apolipoproteins C-II and C-III metabolism in hypertriglyceridemic patients. Effect of a drastic triglyceride reduction by combined diet restriction and fenofibrate administration. Atherosclerosis. 77:139-149.

8. Rees, A., J. Stocks, C. R. Sharpe, M. A. Vella, C. C. Shoulders, J. Katz, N. I. Jowett, F. E. Baralle, and D. J. Galton. 1985. Deoxyribonucleic acid polymorphism in the apolipoprotein A-1-C-III gene cluster. Association with hypertriglyceridemia. J. Clin. Invest. 76:1090-1095.

9. Aalto-Setäla, K., K. Kontula, T. Sane, M. Nieminen, and E. Nikkil. 1987. DNA polymorphisms of apolipoprotein A-I/C-III and insulin genes in familial hypertriglyceridemia and coronary heart disease. Atherosclerosis. 66:145-152.

10. Tas, S. 1989. Strong association of a single nucleotide substitution in the 3 '-untranslated region of the apolipoprotein-CIII gene with common hypertriglyceridemia in Arabs. Clin. Chem. 35:256-259.

11. Ahn, Y. I., R. Valdez, A. P. Reddy, S. A. Cole, K. M. Weiss, and R. E. Ferrell. 1991. DNA polymorphisms of the apolipoprotein AI/CIII/AIV gene cluster influence plasma cholesterol and triglyceride levels in the Mayans of the Yucatan Peninsula, Mexico. Hum. Hered. 41:281-289.

12. Zeng, Q., M. Dammerman, Y. Takada, A. Matsunaga, J. L. Breslow, and J. Sasaki. 1994. An apolipoprotein CIII marker associated with hypertriglyceridemia in Caucasians also confers increased risk in a west Japanese population. Hum. Genet. 95:371-375.

13. Gruber, P. J., A. Torres-Rosado, M. L. Wolak, and T. Leff. 1994. Apo CIII gene transcription is regulated by a cytokine inducible NF-kappa B element. Nucleic Acids Res. 22:2417-2422. 
14. Staels, B., N. Vu-Dac, V. A. Kosykh, R. Saladin, J. C. Fruchart, J. Dallongeville, and J. Auwerx. 1995. Fibrates downregulate apolipoprotein C-III expression independent of induction of peroxisomal acyl coenzyme A oxidase. A potential mechanism for the hypolipidemic action of fibrates. J. Clin. Invest. 95:705-712.

15. Chen, M., J. L. Breslow, W. Li, and T. Leff. 1994. Transcriptional regulation of the apo C-III gene by insulin in diabetic mice: correlation with changes in plasma triglyceride levels. J. Lipid Res. 35:1918-1924.

16. Dammerman, M., L. A. Sandkuijl, J. L. Halaas, W. Chung, and J. L. Breslow. 1993. An apolipoprotein CIII haplotype protective against hypertriglyceridemia is specified by promoter and $3^{\prime}$ untranslated region polymorphisms. Proc. Natl. Acad. Sci. USA. 90:4562-4566.

17. Reue, K., T. Leff, and J. L. Breslow. 1988. Human apolipoprotein CIII gene expression is regulated by positive and negative cis-acting elements and tissue-specific protein factors. J. Biol. Chem. 263:6857-6864.

18. Leff, T., K. Reue, A. Melian, H. Culver, and J. L. Breslow, 1989. A regulatory element in the Apo CIII promoter that directs hepatic specific transcription binds to proteins in expressing and nonexpressing cell types. J. Biol. Chem. 264:16132-16137.

19. Dignam, J. D., R. M. Lebovitz, and R. G. Roeder. 1983. Accurate transcription initiation by RNA polymerase II in a soluble extract from isolated mammalian nuclei. Nucleic Acids Res. 11:1475-1489.

20. Kulozik, A. E., A. Bellan-Koch, S. Bail, E. Kohne, and E. Kleihauer.
1991. Thalassemia intermedia: moderate reduction of beta globin gene transcriptional activity by a novel mutation of the proximal CACCC promoter element. Blood. 77:2054-2058.

21. Smith, J. D., E. A. Brinton, and J. L. Breslow. 1992. Polymorphism in the human apolipoprotein A-I gene promoter region. Association of the minor allele with decreased production rate in vivo and promoter activity in vitro. $J$. Clin. Invest. 89:1796-1800.

22. Reijnen, M. J., F. M. Sladek, R. M. Bertina, and P. H. Reitsma. 1992. Disruption of a binding site for hepatocyte nuclear factor 4 results in hemophilia B Leyden. Proc. Natl. Acad. Sci. USA. 89:6300-6303.

23. Koivisto, U. M. J. J. Palvimo, O. A. Janne, and K. Kontula. 1994. A single-base substitution in the proximal Sp1 site of the human low density lipoprotein receptor promoter as a cause of heterozygous familial hypercholesterolemia. Proc. Natl. Acad. Sci. USA. 91:10526-10530.

24. Reaven, G. M., C. E. Mondon, Y. D. Chen, and J. L. Breslow. 1994. Hypertriglyceridemic mice transgenic for the human apolipoprotein C-III gene are neither insulin resistant nor hyperinsulinemic. J. Lipid Res. 35:820-824.

25. Ogami, K., M. Hadzopoulou-Cladaras, C. Cladaras, and V. I. Zannis. 1990. Promoter elements and factors required for hepatic and intestinal transcription of the human Apo CIII gene. J. Biol. Chem. 265:9808-9815.

26. O'Brien, R. M., M. T. Bonovich, C. D. Forest, and D. K. Granner. 1991. Signal transduction convergence: phorbol esters and insulin inhibit phosphoenolpyruvate carboxykinase gene transcription through the same 10-base-pair sequence. Proc. Natl. Acad. Sci. USA. 88:6580-6584. 